\title{
EQUILIBRIO Y ORGANIZACIÓN DE LA RUTINA DIARIA
}

\section{Silvia Gómez Lillo ${ }^{1}$}

\section{RESUMEN}

Para alcanzar una adecuada calidad de vida es indispensable, entre otros aspectos, organizar en forma conveniente las diversas actividades en que nos involucramos en el diario vivir. En este contexto, el propósito de este artículo es analizar el equilibrio ocupacional partiendo de la revisión del concepto 'adaptación', el que es indispensable para alcanzar, mantenery recuperar este equilibrio de acuerdo a los requisitos de cada cual. El artículo analiza la individualidad del equilibrio, los factores internos y externos que participan, las condiciones que pueden producir desequilibrio y riesgos de no recuperación. Finalmente se mencionan diversos estudios de distribución de actividades diarias y equilibrio ocupacional y algunas medidas que ayudan a alcanzarlo y mantenerlo.

\section{PALABRAS CLAVE}

Desempeño ocupacional, adaptación, equilibrio

\begin{abstract}
In order to get appropriate quality of life is indispensable, between other aspects, to organize in a good way the different activities in which we involving in the daily life.

In this context the purpose of this article is analyze the occupational balance beginning of adaptation concept which is indispensable to obtain and regain this balance accord to everybody require.

The work analyzes the unique balance, the external and internal requirement, and some condition that help to obtain and maintain it.
\end{abstract}

\section{KEYWORDS}

Occupational performance, adaptation, balance.

1 Terapeuta Ocupacional, Licenciada en Ciencia de la Ocupación. Académico Universidad de Chile. Santiago de Chile. E-mail: sgomez@ uchile.cl 


\section{INTRODUCCIÓN}

Para iniciar la discusión en diferentes temas de Ciencia de la Ocupación, relacionados con el desempeño ocupacional, es pertinente comenzar recordando la triada persona-ambiente-ocupación, con el objeto de no perder de vista que los individuos actúan en un medio ambiente que es cambiante, y que se involucran en ocupaciones que son muy variadas.

En este contexto, tenemos que, de esta triada personaambiente-ocupación resulta un desempeño ocupacional que para que sea exitoso es necesario que se combinen adecuadamente múltiples elementos de cada uno de estos componentes. En este artículo nos concentraremos en uno de los temas de estudio de esta ciencia que es el equilibrio de la persona en la organización de sus actividades diarias, en el entendido que éste contribuye a un desempeño ocupacional exitoso que a su vez aporta, en algún grado, a la satisfacción en la vida, y por ende, a su calidad.

Equilibrio es un concepto amplio, que se estudia, y aplica, en diferentes ciencias como lo veremos más adelante. Es así cómo, en un nivel general y también en el que nos ocupa en este artículo, el ámbito ocupacional, para obtener equilibrio es necesario un paso previo, esto es, existir un grado de adaptación, por lo que comenzaremos esta discusión analizando el concepto de adaptación.

\section{AdAPTACIÓN}

En primer término, es importante recordar, que los Terapeutas Ocupacionales usamos el término con diferentes significados como se detalla a continuación.

- Adaptación entendida como cambios en el hogar, los que se aplican generalmente en las viviendas de personas con importantes limitaciones físicas, con el objeto de incrementar su independencia. Algunos ejemplos pueden ser: ubicar la cama en un rincón del dormitorio, con el objeto de asegurar que esta no se desplace ante eventuales contactos bruscos entre las personas y este mueble y para dejar más espacio libre, medida importante en las personas con discapacidades físicas; otros ejemplos son cambiar la altura de los muebles, enchufes y otros; confeccionar rampas de acceso, etc.
- Adaptación como ayuda técnica, también aplicable a persona con alteraciones físicas, con objeto de aumentar su independencia, o su seguridad, en la realización de diversas actividades. Ejemplos de ayudas técnicas pueden ser: modificar engrosando o desviando el mango de la cuchara, con el objeto de que la persona pueda agarrarlo mejor en el primer caso o que pueda ser más fácil acceder a la boca con alimento en el segundo; un vástago con un gancho en un extremo para alcanzar objetos; modificar un cinturón para enganchar un palillo y así la persona pueda tejer con una sola mano; etc.

- Adaptación entendida como ajuste o compensación aplicable a las personas en riesgo o personas con alteraciones que hacen que su relación con el medio ambiente no sea adecuada. Ejemplo de posibles compensaciones puede ser la modificación en el cumplimiento de roles.

En Ciencia de la Ocupación la definición que se considera más precisa y que es la que nos interesa en este artículo es "respuesta interna a un desafío significativo en el desempeño de roles".

De acuerdo a esta definición, en la vida real, no solo las personas en riesgo de adquirir una discapacidad, o personas que la presenten, tienen necesidad de adaptaciones, sino que de hecho todas las personas, continuamente, de distintas formas deben adaptarse en la realización de sus actividades diarias. Antes de analizar cómo y por qué revisaremos definiciones de 'adaptación’ según diversos autores.

La AOTA, en 1979 define el término como "cambio en la función que apoya la supervivencia y la auto actualización" (1), luego agrega que el proceso de adaptación de una persona puede alterarse por condiciones fisiológicas, psicológicas o sociales. Esta definición es amplia y por lo tanto aplicable a las distintas acepciones antes mencionadas.

Dando una mirada hacia atrás podemos ver que el concepto adaptación ha sido considerado importante desde los inicio de la profesión. Así, Meyer, a comienzos del siglo xx, decía que el principal problema que presenta el "paciente" es la "adaptación" y que Terapia Ocupacional "aumentaba las oportunidades de compromiso" de esta persona en las actividades utilizadas 
en la intervención, generalizando luego esta acción a las diversas actividades de su vida diaria.

Avanzando en la historia, recordemos la frase, tan famosa, de Mary Reilly, expresada en la Conferencia Eleanor Clarke de 1966, que dice "El hombre a través del uso de sus manos energizadas por su mente y deseos, puede influenciar el estado de su propia salud". Al analizar esta frase vemos que recalca las premisas de Terapia Ocupacional que plantean que el hombre es un ser activo y que a través de las actividades que realiza puede, de alguna forma, modificar su condición de salud. También está implícito en esta frase que el ser humano al "utilizar sus manos energizadas por su mente y deseos" requiere de un proceso de adaptación. Como el ser humano siempre está "haciendo" (1), la adaptación es un proceso continuo.

Otra autora, Elizabeth Yerxa, luego de su propuesta de creación de la Ciencia de la Ocupación, ha recalcado el significado personal como otra forma de motivarse para ajustarse al medio ambiente correspondiente.

El concepto de adaptación, mirado desde el punto de vista de ajuste o compensación, es muy usado por los terapeutas ocupacionales. Así, si revisamos la literatura sobre el tema encontraremos que continuamente se hace alusión a los siguientes términos, que tienen significado levemente diferente dependiendo del Modelo de Práctica a que estén asociados (1):

- Proceso de adaptación

- Patrones de adaptación

- Funcionamiento adaptativo

- Respuesta adaptativa

- Estrategias adaptativas.

Así, adaptación implica un ajuste al medio ambiente de cada persona, que se produce en forma continua a través del tiempo y que esa persona, en general y en forma inconsciente, trata que sea satisfactorio para ella y para los que la rodean. De esa forma, los ajustes en el contexto que lo estamos analizando, se producen como acciones internas del individuo en el momento que se enfrenta a los desafíos.

Como en nuestro caso, nos interesan los desafíos ocupacionales, vale recordar que en Ciencia de la Ocupación nos referimos a las 'ocupaciones' como las acciones de involucrarse en actividades con propósito, significado y sentido para la persona que la realiza y que estas características se producen porque las personas tienen algún grado de motivación hacia ellas (2). Entonces podemos concluir que las personas deben estar continuamente adaptando su conducta para que, las actividades en las que se involucra, resulten satisfactorias y acordes con el desempeño de sus roles. Así, la adaptación personal se realiza en el momento de la acción pero, por otra parte, las personas deben adaptar estas actividades al tiempo cronológico y orquestarlas de acuerdo a sus necesidades. Más adelante se menciona el concepto 'orquestación' que fue definido por Yerxa en 1990.

El mecanismo de adaptación es un proceso interno que actúa en cada individuo a nivel sub cortical, de acuerdo a las demandas del medio ambiente y que es provocado por las actividades en que nos comprometemos. Adaptarnos a determinada situación nos prepara a situaciones más complejas, a medida que las habilidades requeridas a través de cada adaptación se van internalizando y luego generalizando.

El proceso de adaptación conlleva necesariamente, para su desarrollo, la participación del equilibrio ocupacional

\section{EQUILIBRIO}

El concepto de equilibrio, en las diferentes ciencias que lo incorporan como área de estudio, y para el público, en general, tiene diversas acepciones. Así, por ejemplo, recordemos que 'equilibrio' es una de las materias de estudio de la física que lo define como el estado de un cuerpo solicitado por fuerzas iguales y contrarias, y lo clasifica en equilibrio estático y equilibrio dinámico. De acuerdo a esta definición, en todo cuerpo, y en forma constante, están actuando diferentes tipos de fuerzas, las que ayudan a estos cuerpos a realizar determinados movimientos para mantenerse equilibrados. Lo mismo sucede en la organización de las ocupaciones. Pero, antes de continuar desarrollando el tema recordemos otras acepciones de equilibrio fuera del ámbito de la física. 
- Arreglo armonioso ajustado.

- Comparación o estimación de importancia relativa.

- Ajustes o proporciones de partes simétricas.

- Movimientos con un ritmo en diferentes sentidos.

- Contrapeso, que es el sentido que generalmente se da en Terapia Ocupacional.

En resumen, de lo anterior se desprende que se entiende por equilibrio a igual distribución de elementos con sus características correspondientes, o igual distribución de acciones.

Además de tener diversas acepciones, entre ellas, ser una rama de la Física, de ser objeto de estudio de Ciencia de la Ocupación, el término es usado en otros ámbitos. Así, por ejemplo, en salud se habla de equilibrio hormonal o de la necesidad de equilibrio nutricional; en educación del equilibrio que debe existir entre la entrega académica y la aplicación de situaciones que incrementen la formación personal; los sociólogos mencionan la importancia del equilibrio de las personas entre su dedicación al trabajo y dedicación a la vida familiar, etc.(3)

En lo que a nosotros nos concierne, al igual como sucede con el término 'adaptación', debemos recordar que se ha hablado de la importancia del equilibrio desde los inicios de la profesión. Incluso podríamos decir que es un dogma central que se menciona en las bases filosóficas como agente importante para la mantención de la salud y del bienestar. Adolf Meyer describe la importancia del "equilibrio en de las cuatro extensiones: trabajo y descanso juego y sueño". Este autor opina que debe darse oportunidad a las personas para que organicen su tiempo en rutinas normales de vida, especialmente en el trabajo, dice: "el hombre debe satisfacer sus necesidades interpersonales logrando como resultado bienestar físico y psicológico" (3). Sus principios fueron aplicados en la práctica por Eleanor Clarke, al crear rutinas equilibradas para internos de hospitales psiquiátricos,

Pero el concepto de una vida equilibrada no nace con la Terapia Ocupacional, se usa desde muy antiguo. Así por ejemplo, Shakespeare en Enrique IV dice: "si todos los días fueran feriado hacer deporte sería tan tedioso como trabajar; pero cuando ellos vienen de vez en cuando, ellos se desean".

Mary Reilly en la Conferencia Anual de la AOTA en 1962 dijo, refiriéndose a la importancia del equilibrio ocupacional: "Esta clase de justificación me recuerda la historia de un hombre que murió y despertó rodeado de toda clase de cosas deliciosas que podía obtener con solo mover un dedo. Después que él se satisfizo, llamó al encargado expresándole lo que apreciaba la manera en que había sido tratado y entonces dijo "ahora que yo me he satisfecho deseo hacer algo. Mi buen hombre ¿hay algo para que yo haga en este paraíso?". La respuesta que recibió fue " $\mathrm{Ud}$. lo está haciendo ahora". Pero, replicó nuestro hombre "yo debo hacer algo en mi estadía o mi estadía en el cielo sería intolerable". “¿Quién le dijo a Ud. que estaba en el cielo?". Esta historia puede interpretarse, al igual que la frase de Shakespeare, como la necesidad del ser humano de ocuparse en actividades que le provean distinto significado y propósito, lo que finalmente le da sentido a la vida. Esta autora, en base a su modelo de Conducta Ocupacional, estructuró un programa de rutina diaria que aplicó en un hospital psiquiátrico que incluía actividades que ella llamó de existencia (Actividades de la Vida Diaria), de subsistencia (trabajo y educación) y elegidas (de tiempo libre, incluido el juego), con objeto de restaurar habilidades de sus clientes a través de un patrón equilibrado en la vida diaria.

Kielhofner, tomando como base los autores anteriores propuso el Modelo de Adaptación Temporal con el fundamento que "el uso y organización del tiempo es uno de los indicadores de ajuste exitoso a las demandas de la vida" (1). Con este fundamento, el reafirma lo expresado por Reed y Sanderson quien escribió "equilibrio no implica cantidad de tiempo en cada área, sino que implica algún tiempo en el transcurso de una base regular", que más adelante lo explica diciendo que "el equilibrio más que representar cierta cantidad de trabajo, juego y descanso reflejan una interdependencia dinámica de estos espacios de vida y su relación a valores internos, intereses, metas y para las demandas externas del medio ambiente" (4). Posteriormente estos conceptos fueron incorporados en el subsistema habituación del Modelo de Ocupación Humana (1).

Wilcock dice que el equilibrio es clave entre y con los factores intrínsecos e intrínsecos (5). Csikszentmihaly en la teoría de flujo hace una relación de equilibrio entre habilidades (capacidades) y desafíos (dado en las diferentes acciones, tomando en cuenta las oportunidades para éstas (6). 
Volviendo a lo expuesto por Reed y Sanderson, y por Kielhofner, en nuestro contexto de equilibrio no es el uso de la misma cantidad de tiempo en la realización de actividades, sino el uso del tiempo en el transcurso de una base regular, es necesario considerar que las personas en su vida diaria se comprometen en ocupaciones que son de diversa índole y que estas varían en cantidad, calidad, grado de compromiso y en intensidad a lo largo del tiempo. Así, nos involucramos en distintas ocupaciones de acuerdo a nuestras necesidades, entendiéndose como tal aquellas que están de acuerdo a intereses personales, y de acuerdo a las demandas impuestas por la familia, la sociedad y la cultura (factores externos). Pero, por otra parte, también se hace necesario que las ocupaciones en general, para la calidad del resultado, sean acordes a las capacidades de esfuerzo y a las habilidades de cada cual (factores internos). Finalmente, también es importante que ellas nos den satisfacción (factores internos). Todo lo anterior significa que el equilibrio es incumbencia de cada cual y que su precepción es personal.

Por otra parte, al analizar equilibrio ocupacional, también debemos tomar en cuenta que las áreas de desempeño ocupacional, no se aplican igual para todas las personas. Así por ejemplo, conducir un automóvil puede pertenecer para algunos al área de actividades de la vida diaria, porque implica traslado desde su hogar a su lugar de trabajo, para un chofer de locomoción colectiva significa trabajo remunerado; y para una persona que participa, por ejemplo, en una carrera de regularidad automovilística involucra tiempo libre. Pero, por otro lado, la misma persona puede realizar la ocupación de 'conducir un vehículo', con estos diferentes propósitos en distintos momentos de su vida, incluso en una misma semana. Lo anterior nos muestra que el equilibrio ocupacional es dinámico.

Equilibrio lleva implícito ritmo, o sea, un orden acompasado. Pero, el equilibrio y los ritmos correspondientes, no sólo se dan en las ocupaciones de las personas, se dan en el universo entero. Así, hay equilibrio entre el día y la noche, en los ciclos lunares o en las estaciones del año; hay equilibrio en el funcionamiento de todos los organismos vivos, por ejemplo, en el crecimiento natural de los vegetales o en el funcionamiento de los animales como son el ritmo cardiaco y respiratorio, el ciclo hormonal, nutricional, etc. (3). En todos éstos y también en otras áreas, el equilibrio es un proceso dinámico, que como ya vimos, se da se da a través de la interacción de las partes que lo componen y que están interactuando. Pero hay algunas diferencias, por ejemplo, el equilibrio en el universo es bastante estable, pero el equilibrio ocupacional es inestable ya que está influenciado por el contexto que es extremadamente cambiante, y por el medio en general. Esta inestabilidad es un factor de riesgo que puede afectar positiva o negativamente la salud y el bienestar de las personas.

Si bien, idealmente y en forma continua, debemos tratar de mantener equilibradas nuestras ocupaciones, su mismo dinamismo, factores de personalidad o de condiciones de vida hacen que también haya desequilibrios. De esta manera existe un inter juego continuo entre equilibrio y desequilibrio por lo que algún grado de desequilibrio es normal. Pero si el desequilibrio es mantenido o intenso puede ser nocivo y esto lo vemos especialmente en el área laboral cuando existe sobrecarga o bien gran demanda, situaciones en que la persona se ve apremiada por exigencias que exceden a sus capacidades, o porque deben permanecer largos períodos de tiempo en sus puestos de trabajo y no tienen tiempo para recuperar energía; también observamos sobrecarga en trabajos que se consideran peligrosos, ya sea desde el punto de vista físico o desde el punto de vista mental (7). Lo anterior significa que hay desequilibrio si hay exceso de trabajo, pero también es desequilibrante la falta de éste, ya que si la demanda es baja o la persona tiene pocas oportunidades de trabajo, el desequilibrio producido también es nocivo para la salud. Esta situación se observa tanto en personas desempleadas, como en adultos mayores especialmente los que viven en residencias especiales, o en personas con discapacidades ya sean físicas o mentales.

Así, no siempre se logra adecuado equilibrio en las ocupaciones. Los motivos pueden ser diversos, puede ser por las causas ya mencionadas o porque la selección de lo que nos proponemos realizar, que generalmente es subconsciente, no es la más conveniente, o porque nos vemos obligados a realizarlas por razones sociales. También puede deberse a que no regulamos bien el tiempo que invertimos en cada una de ellas. Otra causa de inadecuada regulación del equilibrio es la mala organización de las secuencias, lo que se traduce en reducida variedad o pobre ordenamiento de las actividades con relación a la intensidad de ellas. Por otra parte, es lógico suponer que el grado de compromiso en las distintas ocupaciones puede variar en el tiempo o en las distintas situaciones, ya sea por interés o por 
necesidad si es que el contexto nos empuja a involucrarnos en determinadas actividades.

En resumen, para organizar las actividades en forma equilibrada deben considerarse factores externos como son los del medio ambiente, tales como obligaciones familiares o sociales, el grado de participación de otros ya sea como colaboradores o como dependientes y las características del medio ambiente en que debemos actuar, que puede ser facilitador o ponernos barreras. También debemos considerar factores internos o personales tales como el interés, capacidad de trabajo, tolerancia, metas auto propuestas; además de edad, sexo, raza. Como es lógico suponer todo lo anterior nos impones necesidades y obligaciones diferentes y cambiantes que no siempre podemos manejar de la forma apropiada.

De acuerdo a lo anterior podemos inferir que es muy probables que no seamos capaces de obtener un equilibrio total, pero lo importante obtener control sobre algunas situaciones de vida y acercarnos lo más posible a él. Esto puede ser difícil, tomemos por ejemplo el caso de una madre que, aunque no lo desee debe acceder a un trabajo remunerado por motivos económicos, no tiene elección; tampoco ella puede, en ésta o en otras de sus ocupaciones decidir la cantidad de tiempo a invertir, así, el trabajo remunerado puede ser de jornada completa, y ser madre no tiene horario, es una ocupación de 24 horas y la energía que se debe invertir suele ser variada y depende de muchos factores. En este contexto, lo más importante como ya se dijo, es tener el mayor control posible sobre las actividades seleccionadas, $y$, por otra parte, estar conscientes sobre nuestras capacidades y los recursos disponibles.

El equilibrio ocupacional tiene repercusiones en la persona y en su medio social. Wilcock plantea que es un factor de riesgo para la salud del individuo y de la comunidad. Esta autora señala que el desequilibrio afecta el funcionamiento de la persona desde el nivel celular hasta las funciones mentales superiores, además de las estructuras ocupacionales de la sociedad y sus políticas (5). Así, las estructuras ocupacionales (escuelas, lugares de trabajo y de esparcimiento de esa comunidad) crean ambientes ocupacionales que afectan en algún grado la salud y el bienestar.

Debido al dinamismo del equilibrio y las constantes variaciones según situaciones diarias, se hace indispensable realizar ajustes permanentemente como ya se ha analizado. En este contexto, no es adecuado para ninguna persona tener un patrón ocupacional totalmente estable ya que, a la larga la rutina diaria sería cansadora y quizás agobiante. Entonces es necesario, como ya se mencionó, tener algún grado de control sobre nuestras elecciones, tomando en cuenta que éstas no sólo impactan en nosotros mismos, sino también en los otros y eventualmente en la sociedad.

\section{ESTUDIOS DE DISTRIBUCIÓN DEL TIEMPO Y EQUILIBRIO OCUPACIONAL}

Los Terapeutas Ocupacionales previos a la formulación de la Ciencia de la Ocupación, demostraron interés en el tema, ya que se realizaron algunos estudios con el objeto de investigar como las personas distribuían su tiempo y organizaban sus ocupaciones, lo que reflejaba de alguna forma el equilibrio ocupacional. En ellas se emplearon distintas metodologías y variables pero los resultados reportados fueron similares. Así, Christiansen, en el año 1977, luego de un análisis de las investigaciones realizadas por Morehead y Takata en el año 1968, Mosey en 1973, Watanabe también en 1973, Cynkin en 1979 y Naville en 1980, concluyó que las personas duermen aproximadamente $30 \%$ de sus vidas, dedican $10 \%$ a Actividades de la Vida Diaria Básicas y $5 \%$ a Actividades de la Vida Diaria Instrumentales, $25 \%$ de su tiempo trabajan o estudian y el $30 \%$ restante lo emplean en actividades de tiempo libre (1).

Posteriormente se han hecho otras investigaciones en la materia. Conocemos estudios realizados en Alemania, Canadá, Australia y Chile. En nuestro país, la primera investigación fue "Patrón de Idiosincrasia de los estudiantes que ingresan al Escuela de Terapia Ocupacional dela Universidad de Chile" y en ella participaron tres promociones a su ingreso. Los resultados fueron similares a los de las investigaciones antes nombradas (2).

Aunque las investigaciones anteriores muestran uso del tiempo, no equilibrio, no dejan de ser de gran importancia ya que de ellas se puede extraer un valioso material relacionado a la organización de las ocupaciones de los grupos estudiados, Además, basándonos en estudios validados de grupos etarios, de especialidad laboral, etc., se puede inferir si personas particulares caen dentro de las normas encontradas en el grupo 
correspondiente y finalmente a partir de ello, es posible que para esa personas analizar el grado de desequilibrio ocupacional de ese momento y estudiar la forma de modificarlo si es necesario.

En investigaciones posteriores realizadas en la Escuela de Terapia Ocupacional de la Universidad de Chile, en adultos mayores y en deportistas destacados $(8,9)$, se analiza en forma tangencial el equilibrio ocupacional.

Si examinamos el concepto de equilibrio desde la perspectiva de la Ciencia de la Ocupación, podemos inferir que no es posible estudiar el equilibrio ocupacional de las personas sólo tomando como referencia el instrumento que en la Universidad de Chile se llama "Patrón de Idiosincrasia" y que fue diseñado a partir del instrumento de S. Cynkin "Configuración Idiosincrática de Actividades" (10), ya que además de representar un corte transversal en la vida de quien se analiza, sólo nos muestra aspectos cuantitativos.

El Patrón de Idiosincrasia muestra la cantidad, la variedad, diversidad de ocupaciones que una persona realiza en un corte transversal en el tiempo, los aspectos temporales, espaciales y sociales, todos estos son datos cuantitativos. Como complemento de lo anterior y para poder deducir el grado de equilibrio, es importante rescatar el propósito, significado y la percepción del uso del tiempo de la persona que es estudiada, durante la ejecución de las distintas ocupaciones de su rutina diaria. En el contexto del Patrón de Idiosincrasia, puede ser el punto de partida para que la persona, a quien le pertenece el patrón realice un exhaustivo análisis, de tipo cualitativo y de acuerdo a características antes señaladas y con la guía del Cientista Ocupacional, o sea Terapeuta Ocupacional especializado en Ciencia de la Ocupación, llegue a resultados relevantes con objeto de hacer modificaciones en orden de mejorar su equilibrio ocupacional. En conclusión en el equilibrio ocupacional es fundamental la percepción personal de su propio hacer y el grado de satisfacción que esto le proporciona.

En la creencia que el equilibrio en las ocupaciones es conveniente para la mantención de la salud y del bienestar, y es un asunto que nos debe interesar tanto desde el punto de vista personal como profesional, deberíamos practicar en nosotros mismos y también en nuestros usuarios algunas medidas para lograrlo. Algunas medidas que se proponen con este fin son las que presentamos a continuación:

Recomendaciones para lograr y mantener el equilibrio ocupacional:

- Analizar detenidamente cuales son las capacidades del involucrado, considerando que las personas nacen con determinadas capacidades que si se practican se transforman en habilidades que, a su vez, pueden convertirse en destrezas. En este contexto, el niño y el adulto joven pueden desarrollar habilidades y destrezas, lo que es más difícil en el adulto mayor, ya que en una etapa más avanzada de la vida la tendencia es hacia una disminución gradual de estas capacidades.

- Luego de analizar las capacidades, el paso siguiente es analizar los recursos con los que se cuenta, sin olvidar que estos pueden ser variables en distintos grados a través del tiempo o de las situaciones del momento.

- Dado que el equilibrio es un proceso dinámico, es necesario re evaluar periódicamente las condiciones anteriores, analizar los beneficios versus costos de las actividades que nos interesa seleccionar, reevaluar intereses, energía invertida, inversión o beneficios económicos, etc., $y$.

- Finalmente, y en estrecha relación con lo anterior, compatibilizar las actividades seleccionadas con los intereses y las necesidades personales principalmente, antes de tomar decisiones.

\section{ConClusión}

Los seres humanos continuamente debemos hacer ajustes en nuestra forma de actuar en la vida diaria con objeto de organizar en forma más satisfactoria y exitosa nuestras rutinas, o sea, hay una continua adaptación. Si la adaptación es adecuada es posible obtener un equilibrio.

Para el logro de equilibrio ocupacional intervienen factores internos y externos que lo hacen un fenómeno complejo que, por esta misma razón es dinámico y también es personal, principalmente debido a los factores internos que intervienen. 
Todo lo anterior hace que el equilibrio ocupacional sea inestable por lo que continuamente las personas caen en pequeños o grandes desequilibrios. Los desequilibrios pasajeros generalmente no son considerados patológicos. Lo importante es mantener control sobre las diversas situaciones de la vida

\section{RefERENCIAS BibLIOGRÁfiCAS}

1. Christiansen C. (1977) Cap.4 Time use and Pattern of Occupations. In Christiansen Ch \& Baum C. (Ed) Occupational Therapy: Enabling, Function \&Well Being. (71-91). Danvers MA. USA. Slack Incorporated.

2. Gómez S., Rueda L., Muñoz C. (2002) "Patrón de Idiosincrasia de los alumnos que ingresan a Terapia Ocupacional". En Bases para el Estudio de la Ciencia de la Ocupación (87-94). Facultad de Medicina. Universidad de Chile.

3. Christiansen Ch. (1996). Three perspectives on balance in occupations. In Zemke R. \& Clark F. (Ed) Occupational Science: an evolving discipline (431-451) Los Ángeles. USA. F.A. Davis Company.

4. Reed K. \& Sanderson S. (1999) Concepts of Occupational Therapy. $4^{\circ}$ Ed. Cap. 8 Concepts related to outcomes and Goals (77-105). Philadelphia. USA. Lippincott Williams \& Wilkins

5. Townsend E., Wilcock A. Occupational Justice. In Christiansen Ch. \& Townsend E. (Ed). Introduction to Occupational Therapy (243-273). New Yersey. USA.

6. Csikzentmihaly M. \& Csikzentmihaly I. S. (Ed). (1988) Optimal Experience psychological studies offlow in consciousness. American Psychological Association. Cambridge University Press.

7. Westrop P. (3003) Balance Exploring as concept in occupation. Journal of Occupational Science 10 (2) 99-106.

8. Gómez S. (2002) Percepción de los cambios en el Desempeño Ocupacional frente a la situación de Jubilación de Chilenos que viven en Suecia. Revista Chilena de Terapia Ocupacional N ${ }^{\circ}$ 2 (22-25).

9. Capel A. \& Rojas D (2006) Estudio del Perfil Ocupacional de Deportistas PRO-DAR concentrados en el Centro de Alto Rendimiento de Santiago de Chile, (tesis de pe grado para optar al título de Terapeuta Ocupacional. Facultad de Medicina. Universidad de Chile. Santiago, Chile.

10. Cynkin S., Mazur A. (1989). Occupational Therapy and Activities Health: Toward health Through Activities. Appendix A Idiosyncratic Activities Configuration- Instructions and Questionnaire (273-276) Boston. Editorial Little Brown 